\title{
Electrochemical Construction of Low-crystalline CoOOH with Short-range Ordered Grains to Improve Oxygen Evolution Activity
}

Shenghua Ye, ${ }^{\mathrm{a}, \mathrm{b}}$ \# Jingpeng Wang, ${ }^{\mathrm{a}}$ J Jing Hu, ${ }^{\mathrm{a}}$ \# Zhida Chen, ${ }^{\mathrm{a}}$ Lirong Zheng, ${ }^{\mathrm{c}}$ Yonghuan Fu, ${ }^{\mathrm{a}}$ Yaqi Lei, ${ }^{\mathrm{a}}$ Xiangzhong Ren, ${ }^{\mathrm{a}}$ Chuanxin He, ${ }^{a}$ Qianling Zhang*a and Jianhong Liu*a,b

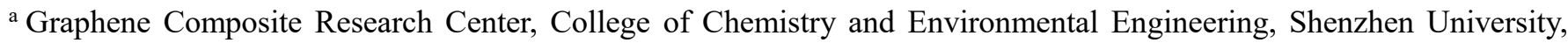
Shenzhen, 518060, P. R. China,

${ }^{\mathrm{b}}$ Shenzhen Eigen-Equation Graphene Technology Co. Ltd., Shenzhen, 518000, P. R. China;

${ }^{\mathrm{c}}$ Institute of High Energy Physics Chinese Academy of Sciences, Beijing, 100049, PR China

*Corresponding author

E-mail: zhq1@szu.edu.cn; liujh@szu.edu.cn;

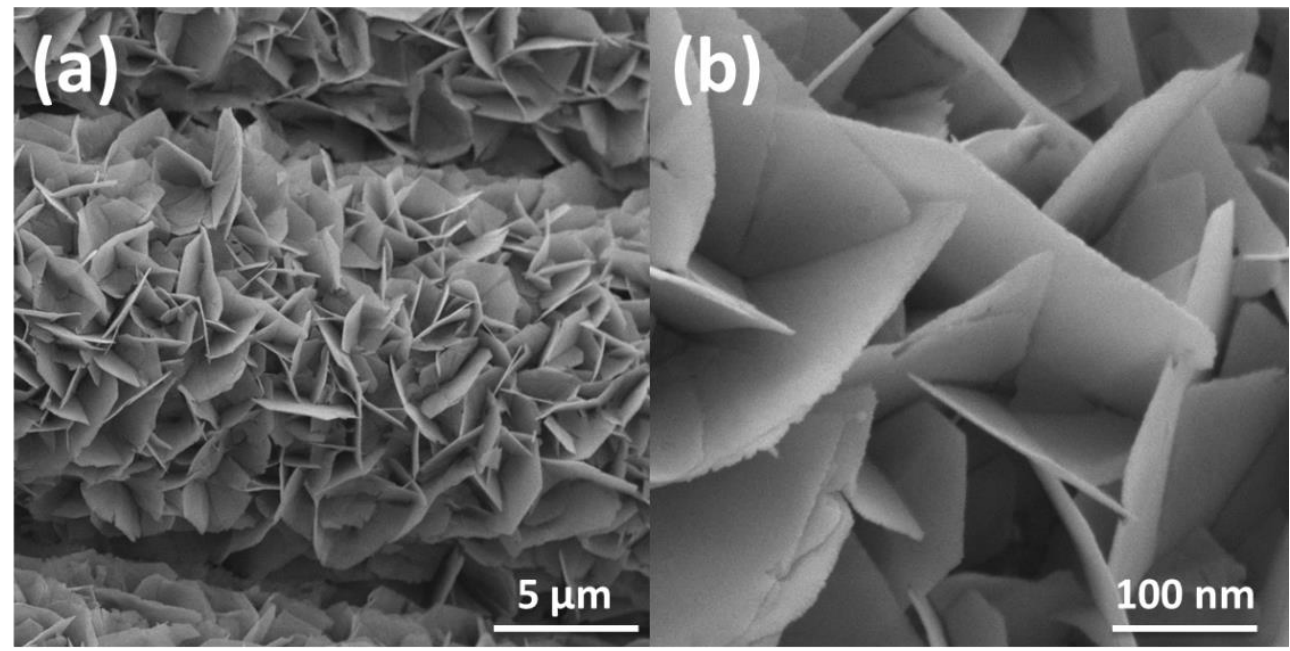

Figure S1 SEM images of $\mathrm{Co}(\mathrm{OH})_{2} \mathrm{NAs} / \mathrm{CFC}$.

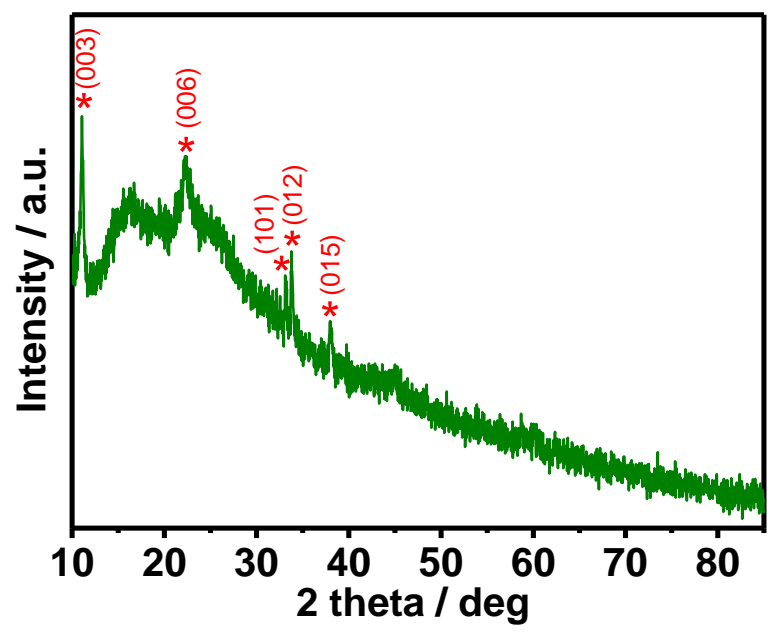

Figure S2 XRD pattern of $\mathrm{Co}(\mathrm{OH})_{2} \mathrm{NAs} / \mathrm{CFC}(\mathrm{JCPDS}-\# 74-1057)$. 


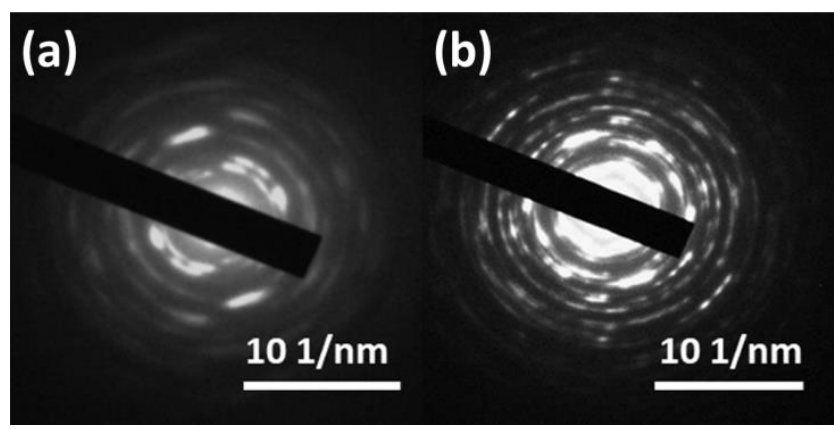

Figure S3 SAED patterns of (a) LC-CoOOH NAs/CFC and (b) CoOOH NAs/CFC.
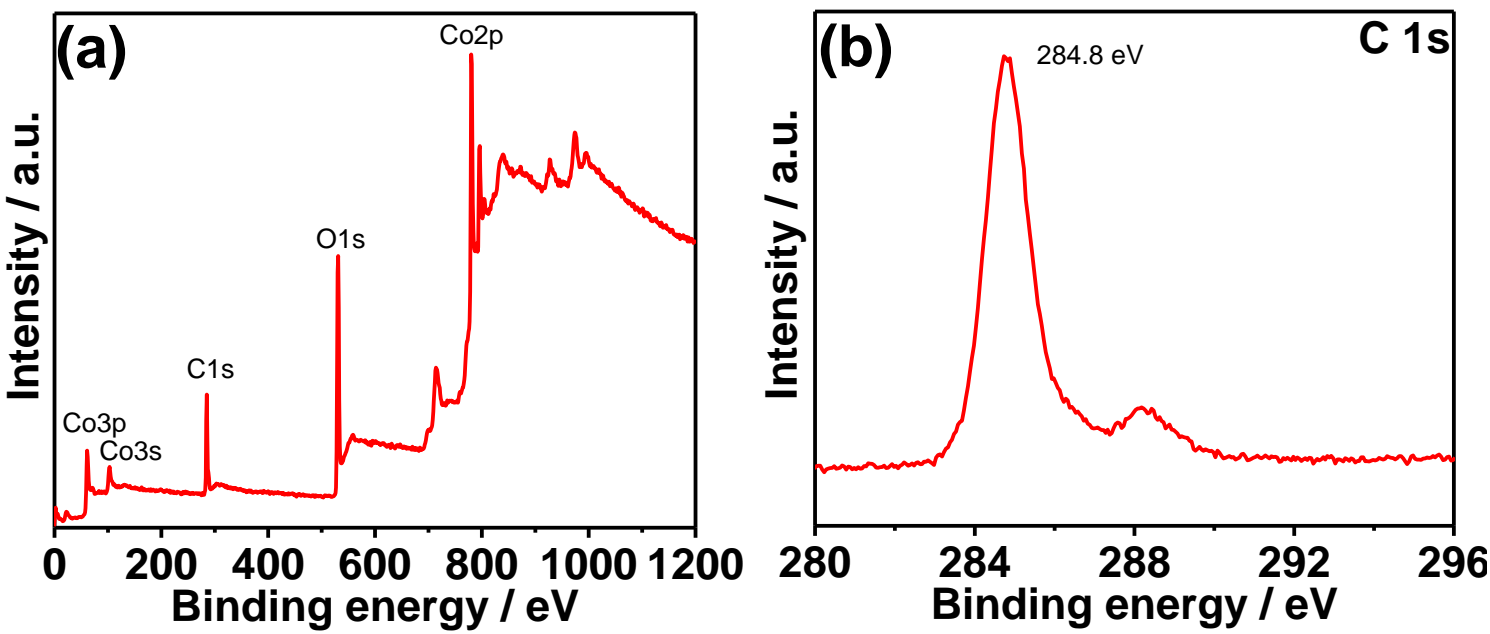

Figure S4 XPS of (a) survey and (b) C1s of LC-CoOOH NAs/CFC.
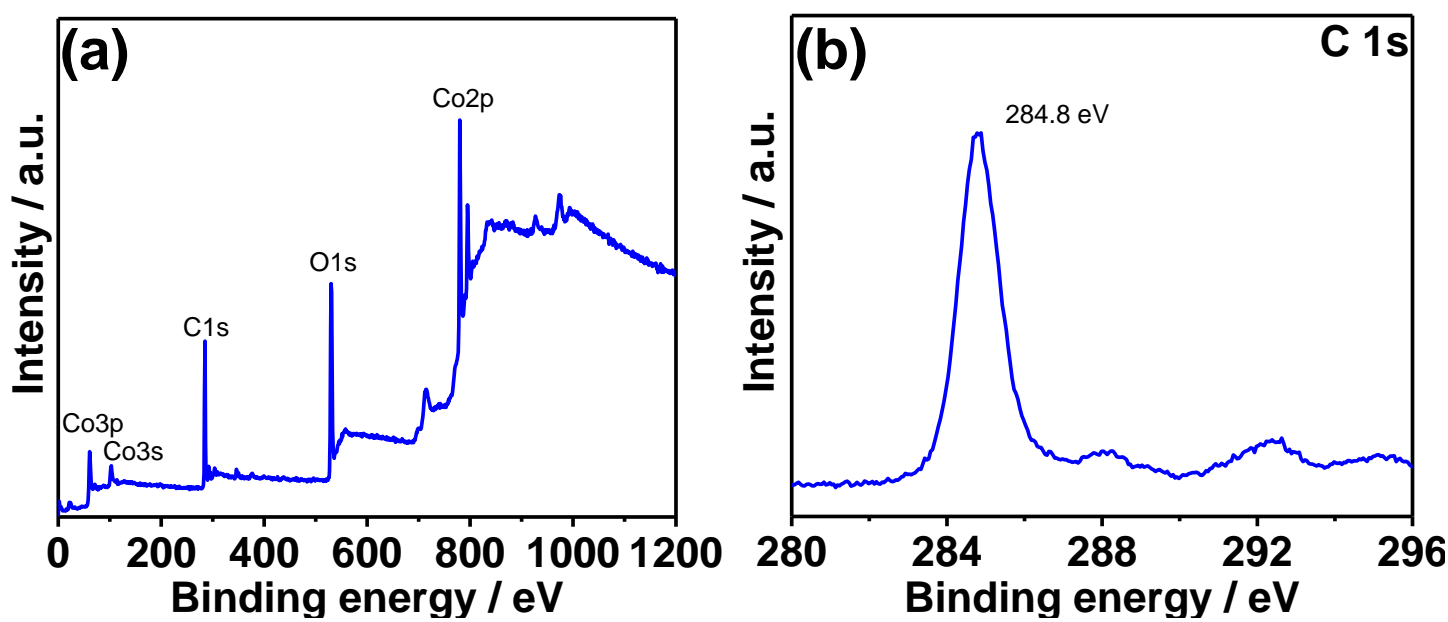

Figure S5 XPS of (a) survey and (b) $\mathrm{C} 1 \mathrm{~s}$ of $\mathrm{CoOOH} \mathrm{NAs} / \mathrm{CFC}$. 

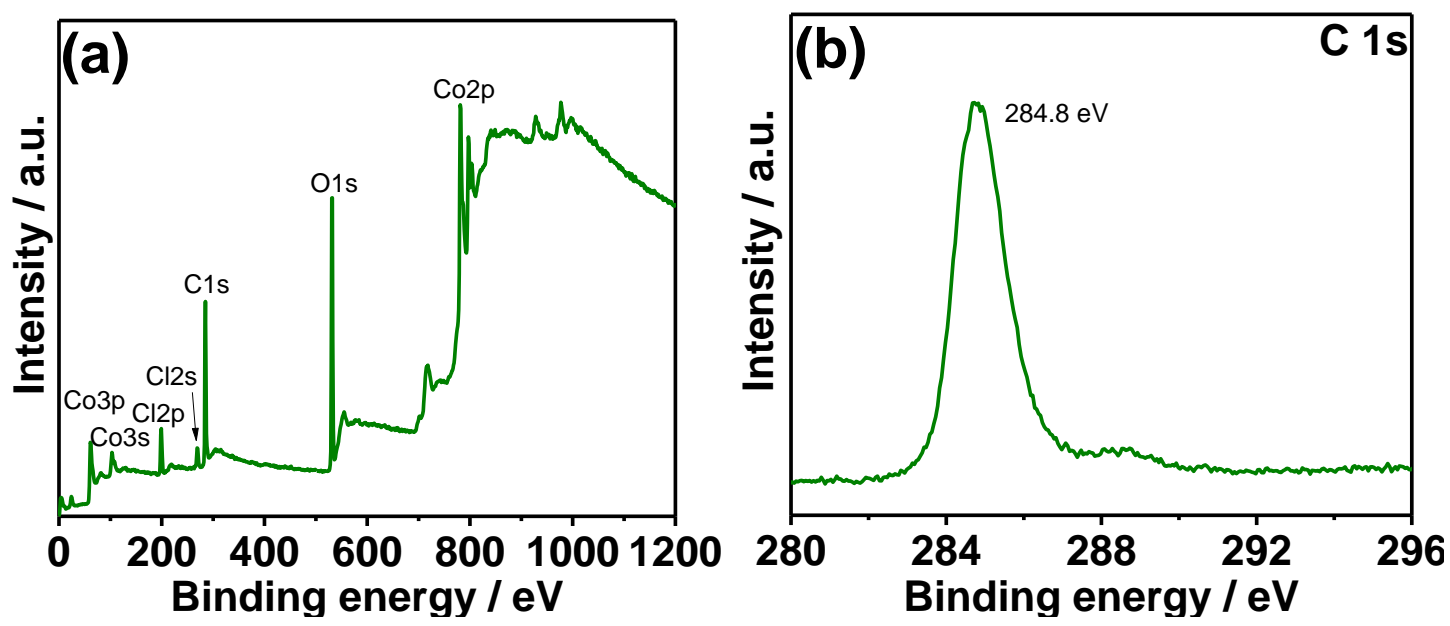

Figure S6 XPS of (a) survey and (b) $\mathrm{C} 1 \mathrm{~s}$ of $\alpha-\mathrm{Co}(\mathrm{OH})_{2} \mathrm{NAs} / \mathrm{CFC}$.

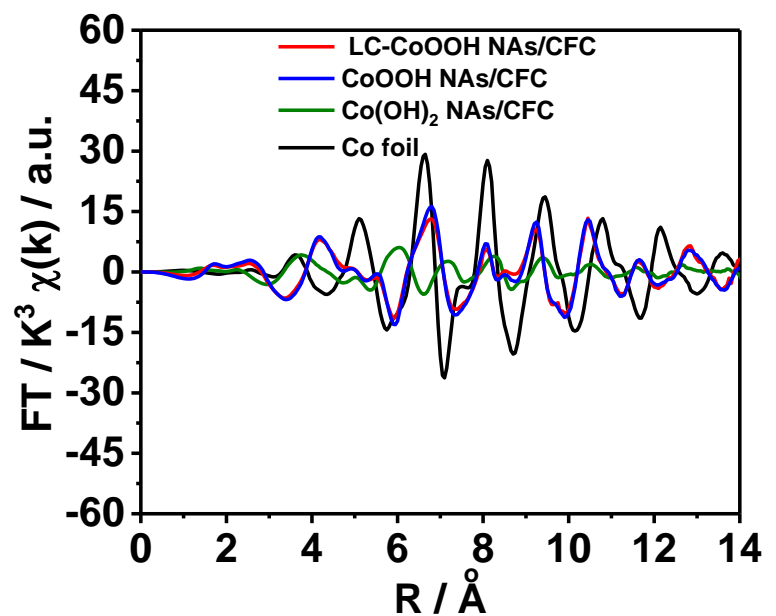

Figure S7 EXAFS of LC-CoOOH NAs/CFC, $\mathrm{CoOOH} \mathrm{NAs/CFC,} \mathrm{Co}(\mathrm{OH})_{2} \mathrm{NAs} / \mathrm{CFC}$, and Co foil.

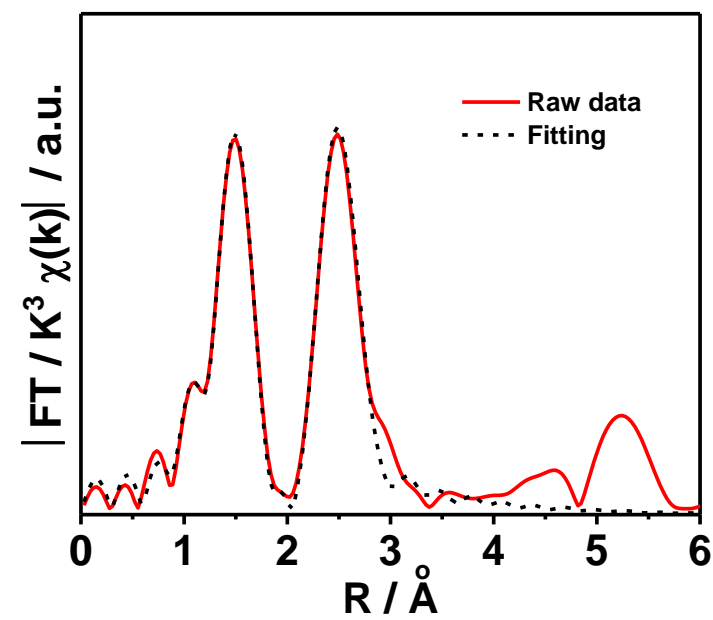

Figure S8 EXAFS fitting of LC-CoOOH NAs/CFC. 
Table S1. EXAFS fitting parameters at the Co K-edge for various samples

\begin{tabular}{ccccccc}
\hline Sample & Shell & $\boldsymbol{N}^{a}$ & $\boldsymbol{R}\left(\AA^{\boldsymbol{a}}\right)^{\boldsymbol{b}}$ & $\boldsymbol{\sigma}^{\mathbf{2}}\left(\AA^{\left.\mathbf{2} \cdot \mathbf{1 0}^{\mathbf{3}}\right)^{\boldsymbol{c}}}\right.$ & $\boldsymbol{\Delta \boldsymbol { E } _ { \mathbf { 0 } } ( \mathbf { e V } ) ^ { \boldsymbol { d } }}$ & $\boldsymbol{R}$ factor $(\mathbf{\%})$ \\
\hline LC- & Co-O & 5.2 & 1.90 & 2.8 & 2.9 & 0.6 \\
CoOOH & Co-Co & 4.2 & 2.84 & 3.4 & -4.8 & \\
NAs/CFC & & & & & & \\
CoOOH & Co-O & 6 & 1.90 & 4.0 & 3.5 & 0.6 \\
NAs/CFC & Co-Co & 6 & 2.84 & 4.6 & -4.6 & \\
\hline
\end{tabular}

${ }^{a} N$ : coordination number; ${ }^{b} R$ : bond distance; ${ }^{c} \sigma^{2}$ : Debye-Waller factor; ${ }^{d} \Delta E_{0}$ : the inner potential correction. $R$ factor: goodness of fit. $S_{0}^{2}, 0.872$, was obtained from the experimental EXAFS fit of the $\mathrm{CoOOH}$ reference by fixing $\mathrm{CN}$ as the known crystallographic value and was fixed to all the samples.
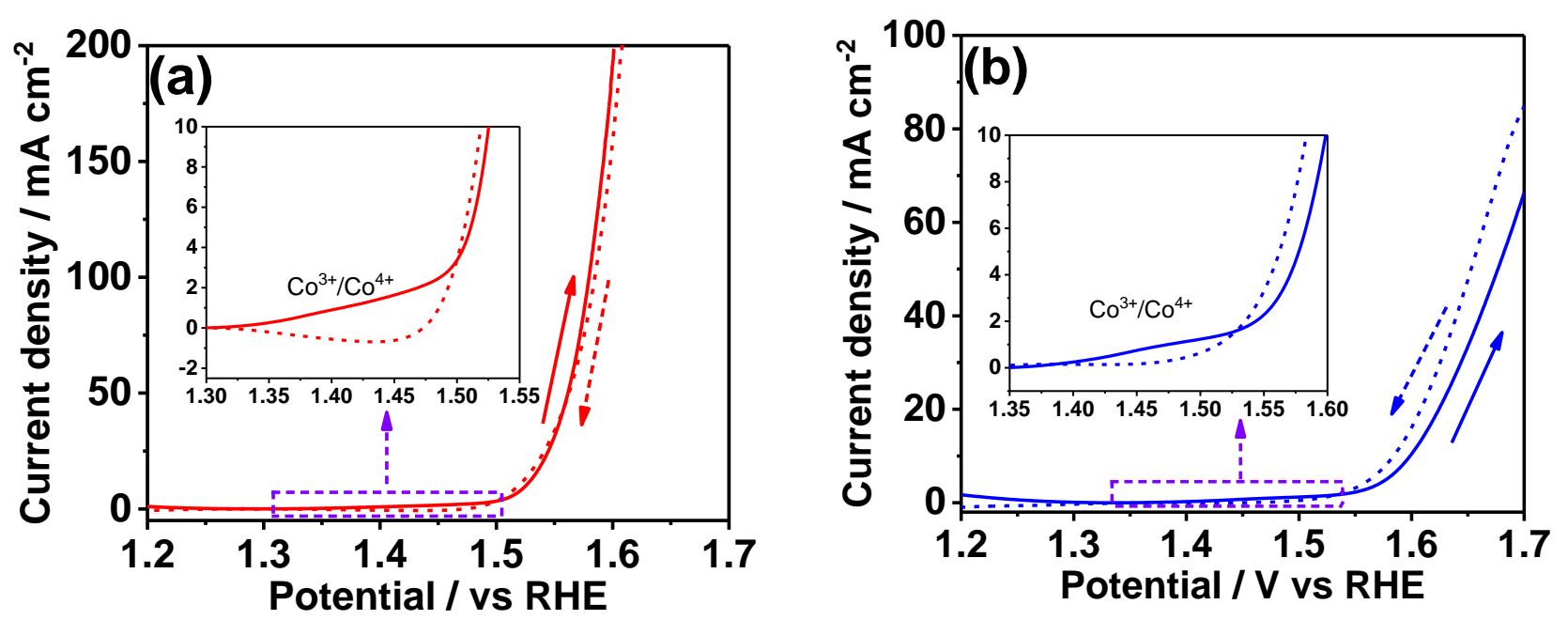

Figure S9 LSV curves of (a) LC-CoOOH NAs/CFC and (b) $\mathrm{CoOOH} \mathrm{NAs/CFC} \mathrm{with} \mathrm{forward} \mathrm{and} \mathrm{reverse} \mathrm{scan} \mathrm{at} 5$ $\mathrm{mV} / \mathrm{s}$. 

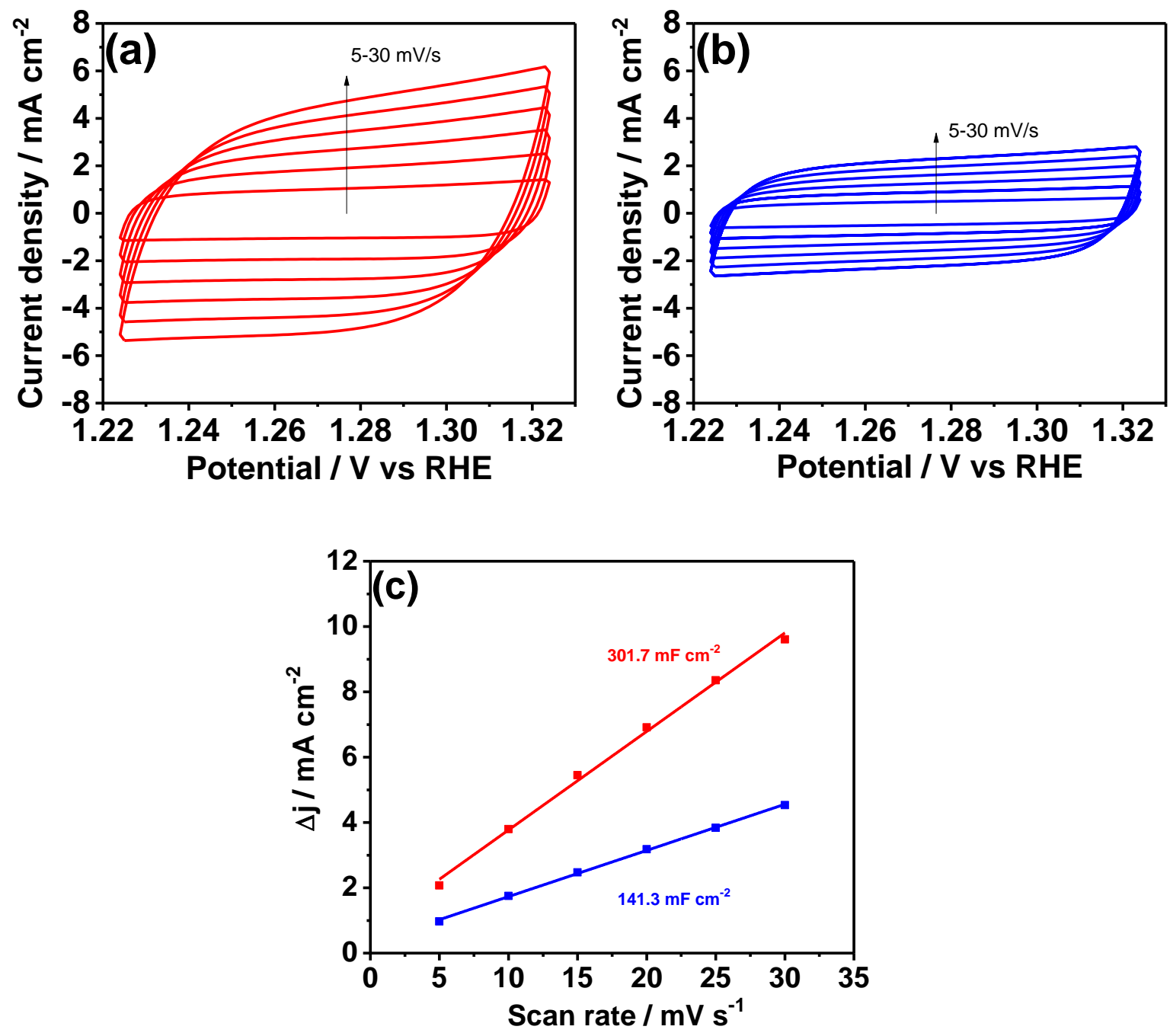

Figure S10 CV curves of (a) LC-CoOOH NAs/CFC; (b) CoOOH NAs/CFC, and (c) CFC; (d) current density variation plotted against the scan rate, fitted to a linear regression, which enables the estimation of the double-layer capacitance $\left(\mathrm{C}_{\mathrm{dl}}\right)$.

The electrochemically active areas (ECSAs) of LC-CoOOH NAs/CFC and CoOOH NAs/CFC were determined by the formula

$$
\mathrm{R}_{\mathrm{f}}=\frac{\mathrm{C}_{\mathrm{dl}}}{60}
$$

in which the $\mathrm{C}_{\mathrm{dl}}$ of samples were deduced by the value of the CFC; the $\mathrm{C}_{\mathrm{dl}}$ of an ideal unit area was assumed to be $60 \mu \mathrm{F}$ $\mathrm{cm}^{-2} . \mathrm{R}_{\mathrm{f}}$ is the roughness factor.

The electrochemical active area (ECSA) was calculated by the formula

$$
E C S A=R_{\mathrm{f}} \times A
$$

where $\mathrm{A}$ is the surface area of the electrode. ${ }^{1}$ 


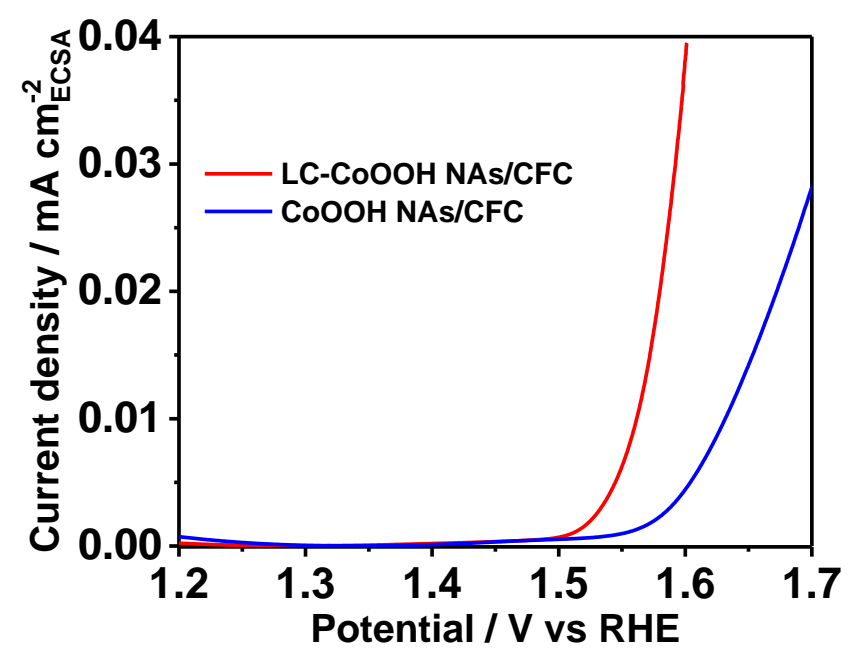

Figure S11 LSV curves of LC-CoOOH NAs/CFC and CoOOH NAs/CFC, with the current density normalized to the ECSA

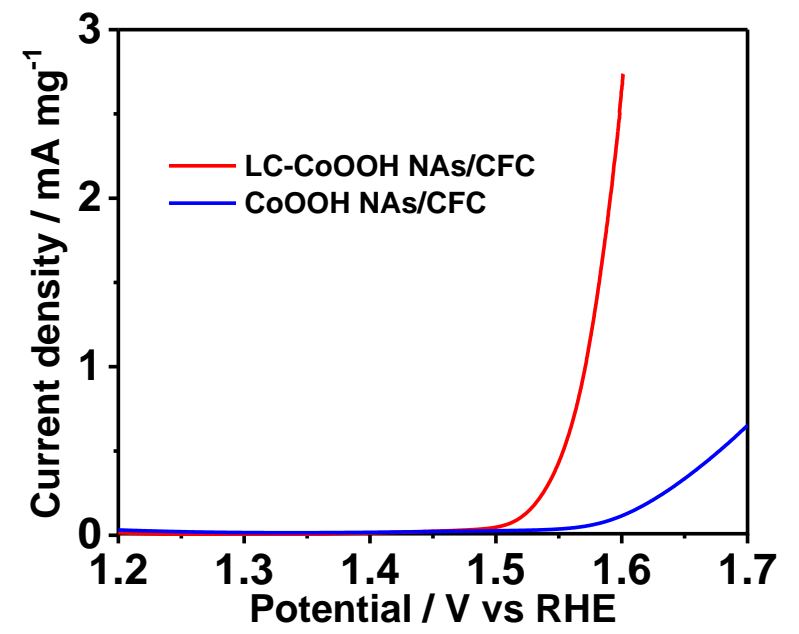

Figure S12 LSV curves of (a) LC-CoOOH NAs/CFC and (b) CoOOH NAs/CFC normalized to the mass of CoOOH.

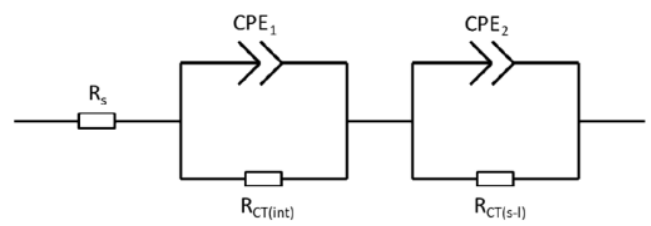

Figure S13 Simplified equivalent circuit model of EIS.

Table S2 Fitting parameters of EIS

\begin{tabular}{cccccc}
\hline Sample & CPE $_{\mathbf{1}}$ & $\mathbf{C P E}_{\mathbf{2}}$ & $\mathbf{R}_{\mathbf{s}}$ & $\mathbf{R}_{\mathbf{C T}(\mathrm{int})}$ & $\mathbf{R}_{\mathrm{CT}(\mathrm{s}-\mathrm{I})}$ \\
\hline LC-CoOOH NAs/CFC & 0.00009 & 0.1743 & 4.723 & 2.050 & 4.930 \\
CoOOH NAs/CFC & 0.00016 & 0.1019 & 3.218 & 3.263 & 11.07 \\
\hline
\end{tabular}



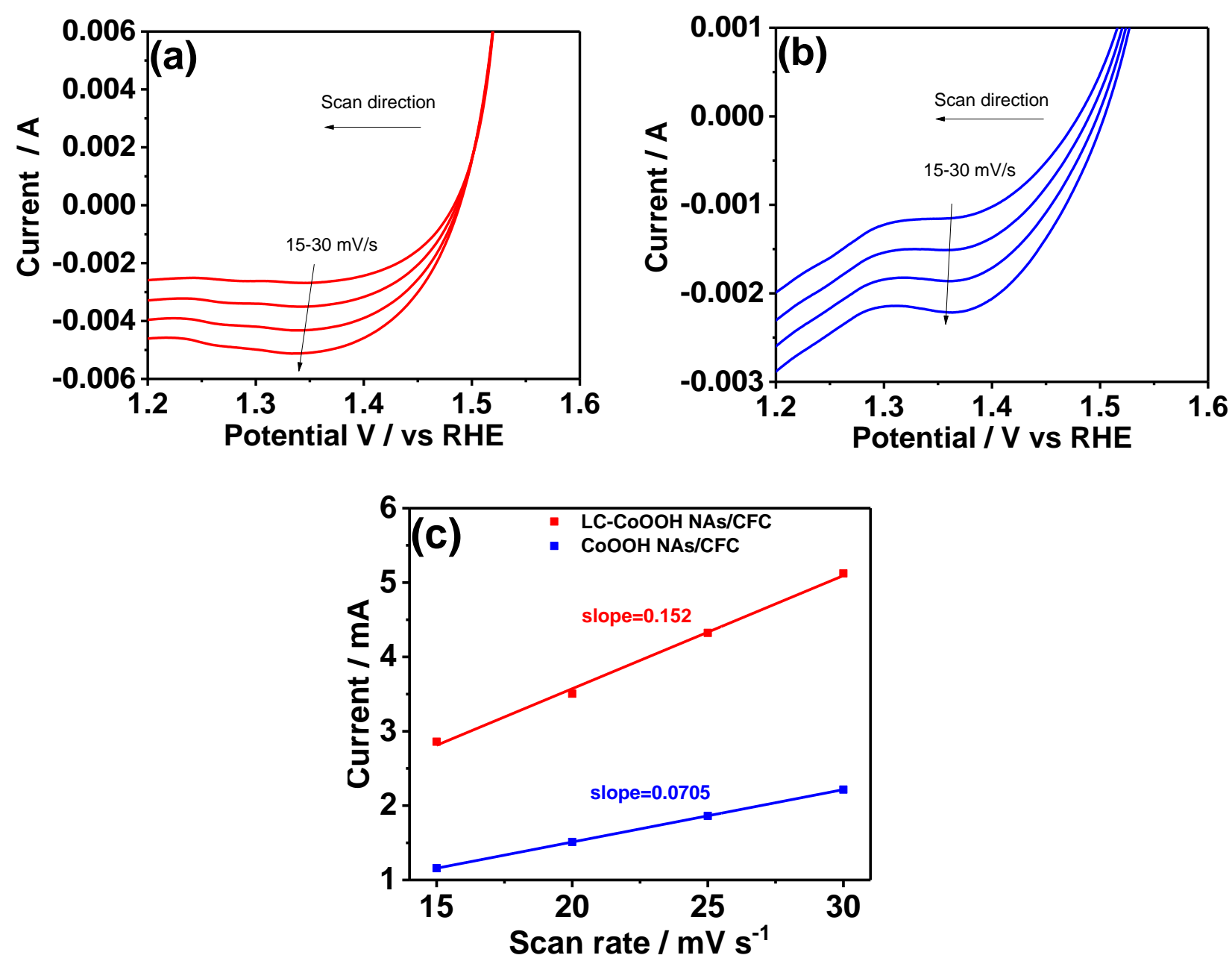

Figure S14 CVs for (a) LC-CoOOH NAs/CFC and (b) CoOOH NAs/CFC under different scan rates from 10 to $60 \mathrm{mV}$ $\mathrm{s}^{-1}$ in $1.0 \mathrm{M} \mathrm{KOH}$; (c) linear relationship of the peak currents vs. scan rates for (a) LC-CoOOH NAs/CFC and (b) $\mathrm{CoOOH} \mathrm{NAs} / \mathrm{CFC}$ at different fixed overpotentials.

The number of surface active sites associated with the redox Co species should first be calculated. The linear relationship between the oxidation peak current and the scan rate can be extracted from the electrochemical cyclic voltammetry scans.

The slope of the line can be calculated based on the following equation:

$$
\text { slope }=\frac{n^{2} F^{2} m}{4 R T}
$$

in which $\mathrm{n}$ is the number of electrons transferred in the redox of $\mathrm{Co}^{3+} / \mathrm{Co}^{4+}, \mathrm{F}$ is the Faraday constant, $\mathrm{m}$ is the number of active sites (mol), and $\mathrm{R}$ and $\mathrm{T}$ are the ideal gas constant and the absolute temperature, respectively. ${ }^{2}$ 


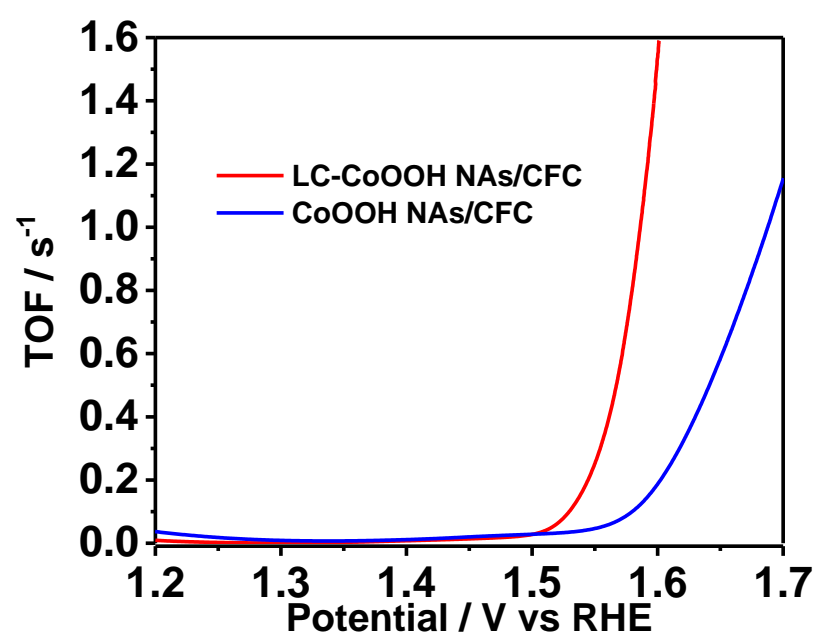

Figure S15 TOF curves of LC-CoOOH NAs/CFC and CoOOH NAs/CFC.

The TOF value can be finally calculated using the formula

$$
\mathrm{TOF}=\frac{J A}{4 F m}
$$

in which the TOF is based on the number of redox-active sites, $\mathrm{J}$ is the current density at a certain overpotential, $\mathrm{A}$ is the area of the electrode, 4 indicates the moles of electrons consumed to evolve one mole of $\mathrm{O}_{2}$ from $\mathrm{H}_{2} \mathrm{O}, \mathrm{F}$ is the Faraday constant, and $\mathrm{m}$ is the number of moles of the active sites. ${ }^{2}$ 


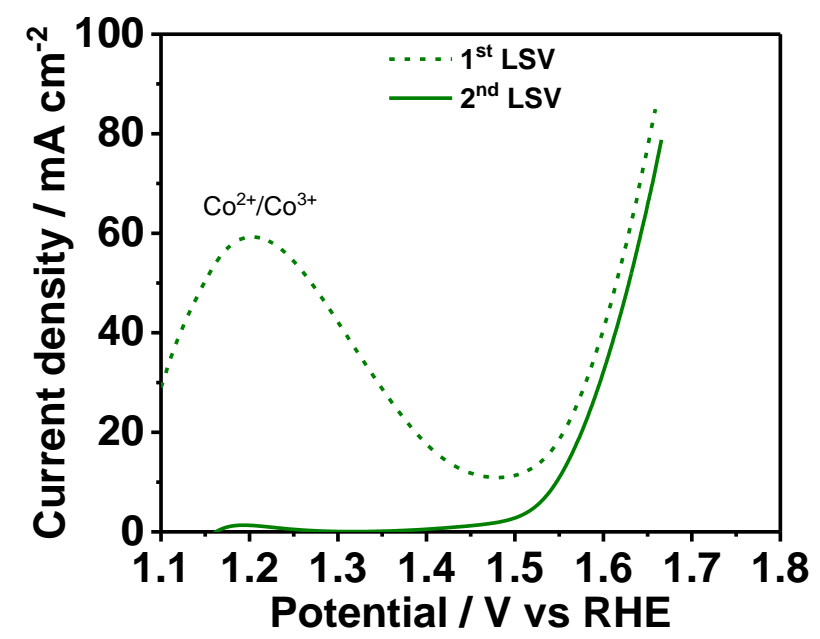

Figure S16 LSV curves of $\mathrm{Co}(\mathrm{OH})_{2} \mathrm{NAs} / \mathrm{CFC}$.

As shown in Figure S16, there is a large redox peak before onset of OER current at the first scan. However, this redox peak disappeared at the second scan. This phenomenon indicates that there is an irreversible oxidation occurred at the first scan. The $\mathrm{Co}^{2+}$ in $\mathrm{Co}(\mathrm{OH})_{2}$ has been irreversibly oxidized to $\mathrm{Co}^{3+}$. The catalytic activity of $\mathrm{Co}(\mathrm{OH})_{2} \mathrm{NAs} / \mathrm{CFC}$ is still inferior than that of LC-CoOOH NAs/CFC, implying $\mathrm{Co}(\mathrm{OH})_{2}$ may had been oxidized to another inactive phase of $\mathrm{CoOOH}$. Therefore, this result highlights that the proper oxidation strategy (in situ anodic oxidation) is crucial for the catalytic performance.

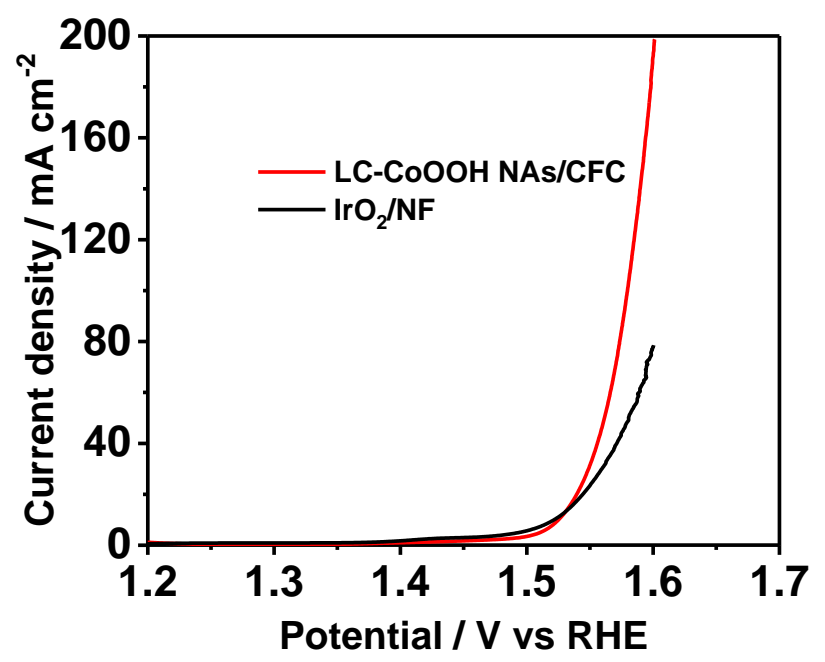

Figure S17 LSV curves of LC-CoOOH NAs/CFC and $\mathrm{IrO}_{2} / \mathrm{NF}$. 

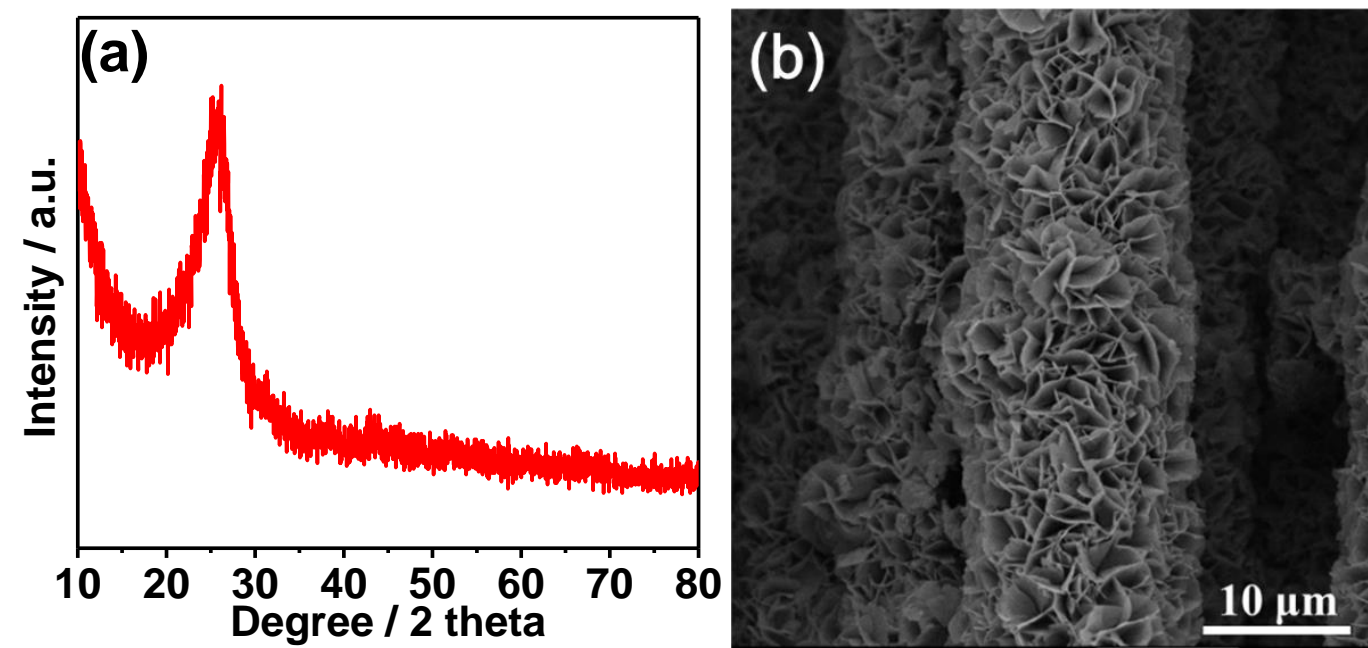

Figure S18 (a) XRD pattern and (b) SEM image of LC-CoOOH NAs/CFC after the durability test.

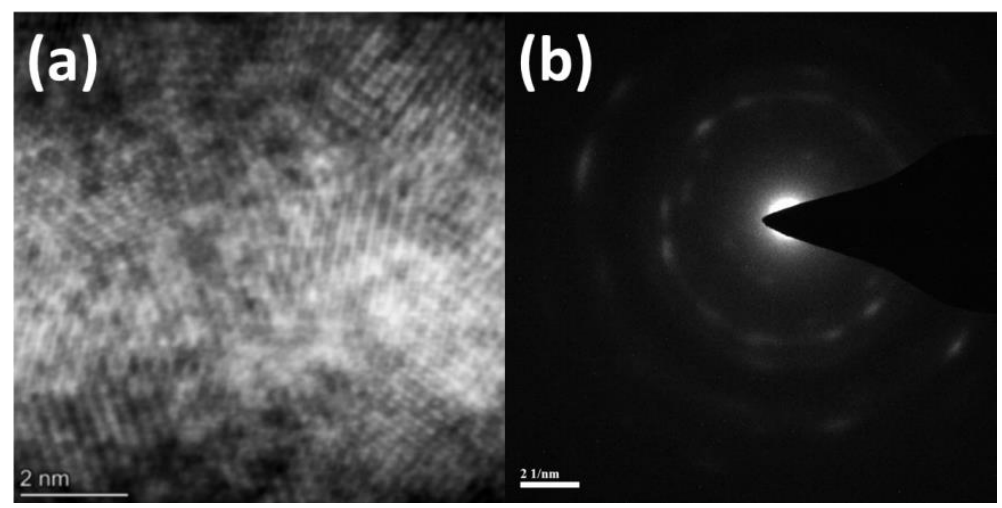

Figure S19 iDPC-STEM image and corresponding SAED pattern of LC-CoOOH NAs/CFC after the durability test. 

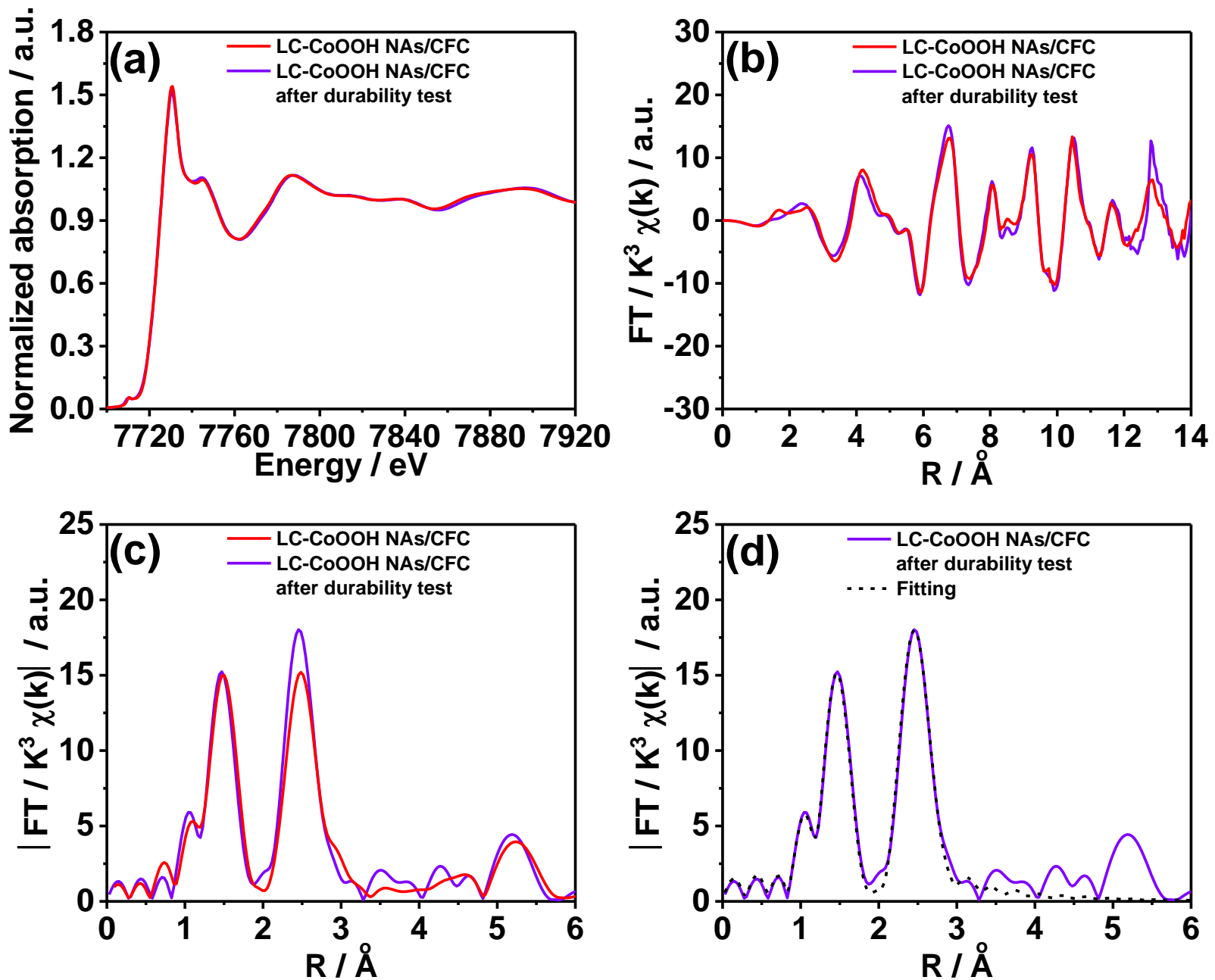

Figure S20 (a) XANES, (b) EXAFS and (c) FT-EXAFS of Co K-edge of LC-CoOOH NAs/CFC and LC-CoOOH NAs/CFC after the durability test. (d) Fitting curves of T-EXAFS of Co K-edge of LC-CoOOH NAs/CFC after the durability test.

Table S3 EXAFS fitting parameters at the Co K-edge for various samples

\begin{tabular}{|c|c|c|c|c|c|c|c|}
\hline \multicolumn{2}{|c|}{ Sample } & Shell & $N^{a}$ & $R(\AA)^{b}$ & $\sigma^{2}\left(\AA^{2} \cdot 10^{3}\right)^{c}$ & $\Delta E_{0}(\mathrm{eV})^{d}$ & $R$ factor $(\%)$ \\
\hline \multirow[t]{2}{*}{ LC-CoOOH } & NAs/CFC & $\mathrm{Co}-\mathrm{O}$ & 5.2 & 1.90 & 2.8 & 2.9 & 0.6 \\
\hline & & $\mathrm{Co}-\mathrm{Co}$ & 4.2 & 2.84 & 3.4 & -4.8 & \\
\hline LC-CoOOH & NAs/CFC & $\mathrm{Co}-\mathrm{O}$ & 5.4 & 1.89 & 3.3 & -0.3 & 0.5 \\
\hline \multicolumn{2}{|c|}{ after durability test } & $\mathrm{Co}-\mathrm{Co}$ & 4.2 & 2.82 & 3.2 & -8.2 & \\
\hline
\end{tabular}

${ }^{a} N$ : coordination number; ${ }^{b} R$ : bond distance; ${ }^{c} \sigma^{2}$ : Debye-Waller factor; ${ }^{d} \Delta E_{0}$ : the inner potential correction. $R$ factor: goodness of fit. $S_{0}^{2}, 0.872$, was obtained from the experimental EXAFS fit of the $\mathrm{CoOOH}$ reference by fixing $\mathrm{CN}$ as the known crystallographic value and was fixed to all the samples. 

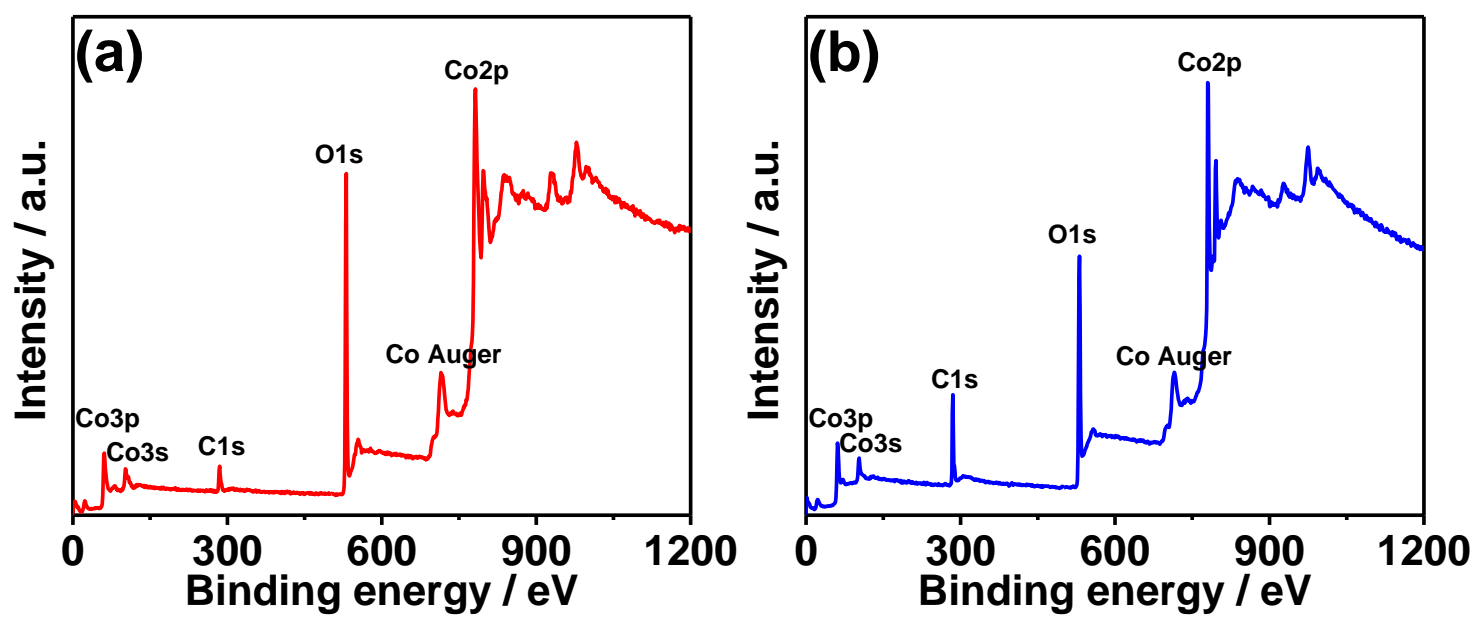

Figure S21 XPS spectra of (a) LC-CoOOH NAs/CFC and (b) CoOOH NAs/CFC after durability test.

The signal of Fe $2 p$ could not be found except Co Auger peak in the survey of LC-CoOOH NAs/CFC and $\mathrm{CoOOH}$ NAs/CFC (Figure S21), suggesting that there isn't obvious impurity Fe in both LC-CoOOH $\mathrm{NAs} / \mathrm{CFC}$ and $\mathrm{CoOOH} \mathrm{NAs} / \mathrm{CFC}$ after durability test.

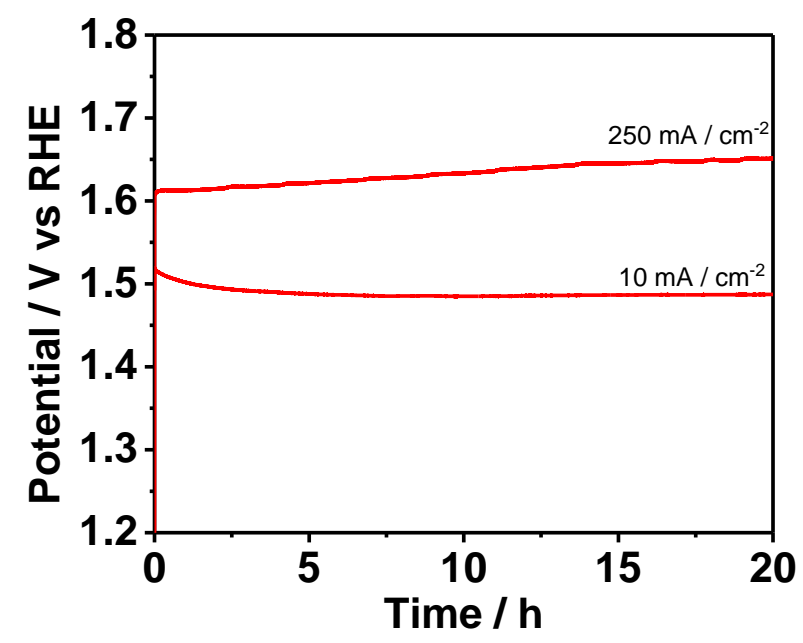

Figure S22 E-t curves of LC-CoOOH NAs/CFC under different current densities. 
(a)
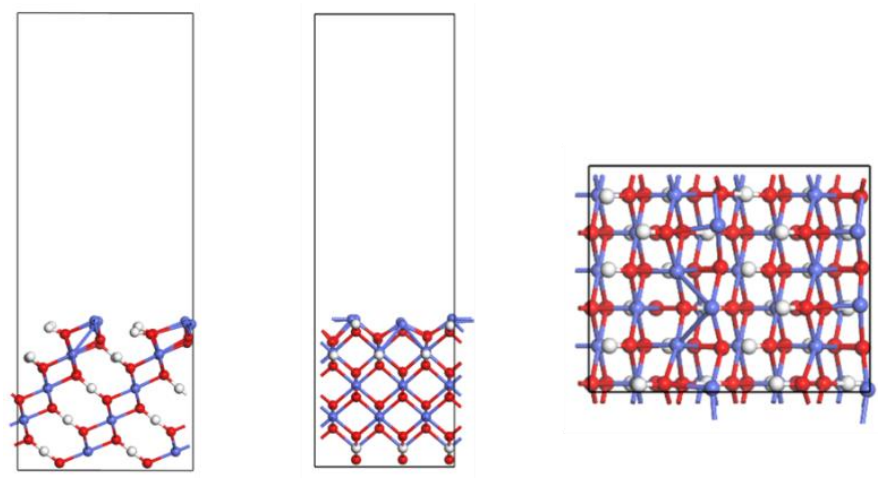

(b)
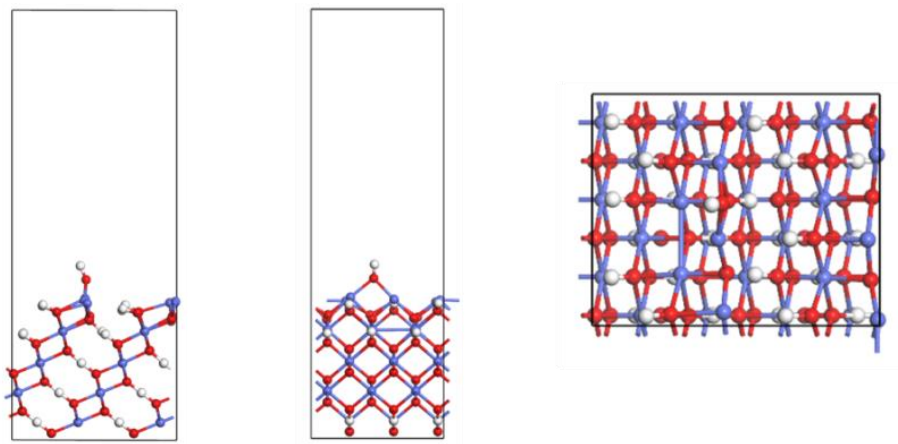

(c)
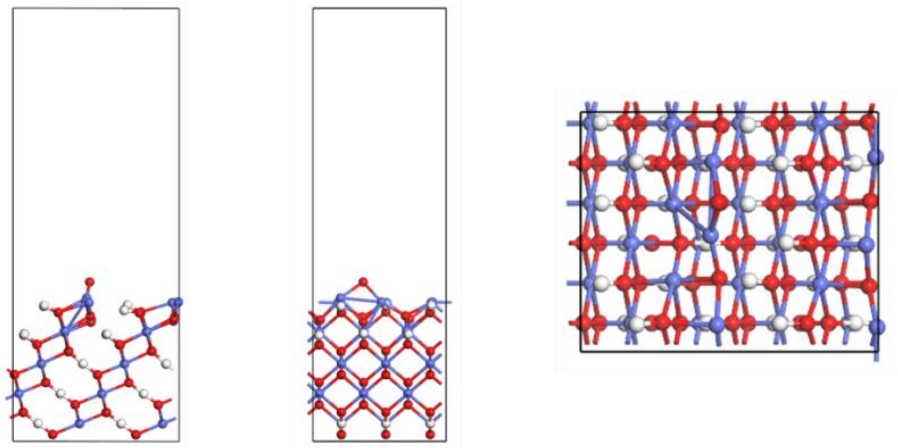

(d)
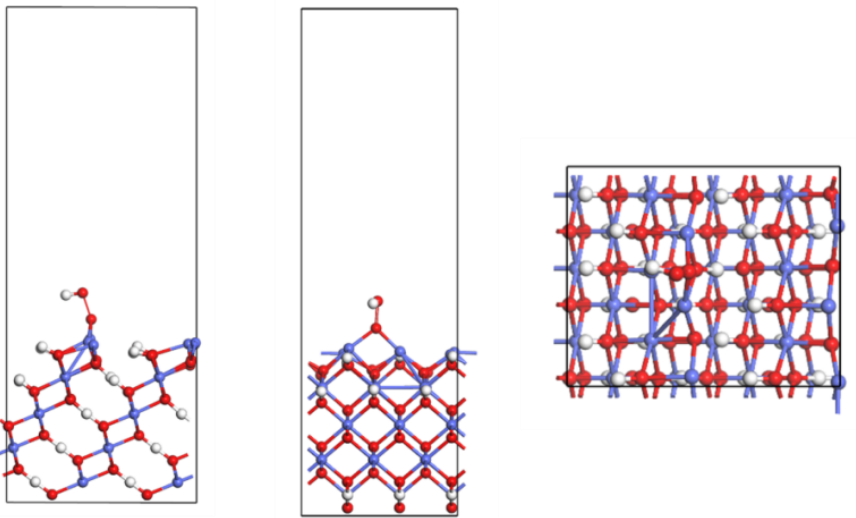

Figure S23 Optimized configurations of (a) (104) of $\mathrm{CoOOH}$ with oxygen vacancies and (b) $\mathrm{OH}^{*}$, (c) $\mathrm{O}^{*}$, and (d) $\mathrm{OOH}^{*}$ species adsorbed on (104) of $\mathrm{CoOOH}$ with oxygen vacancies. 
(a)
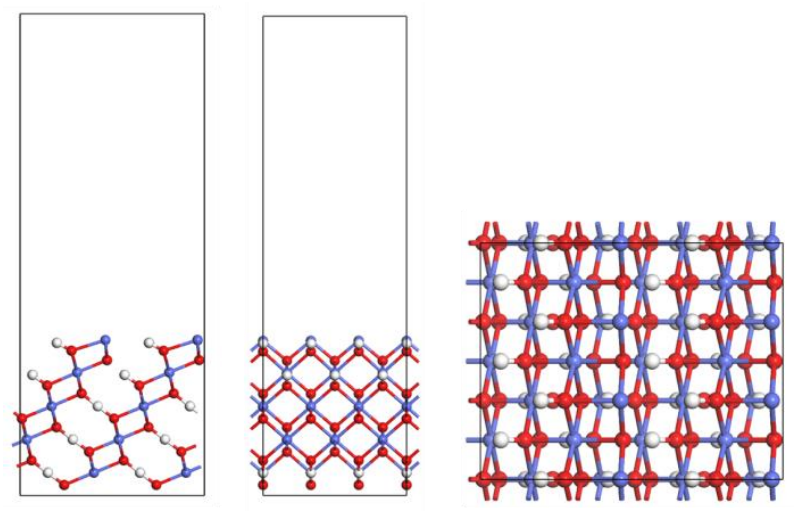

(b)
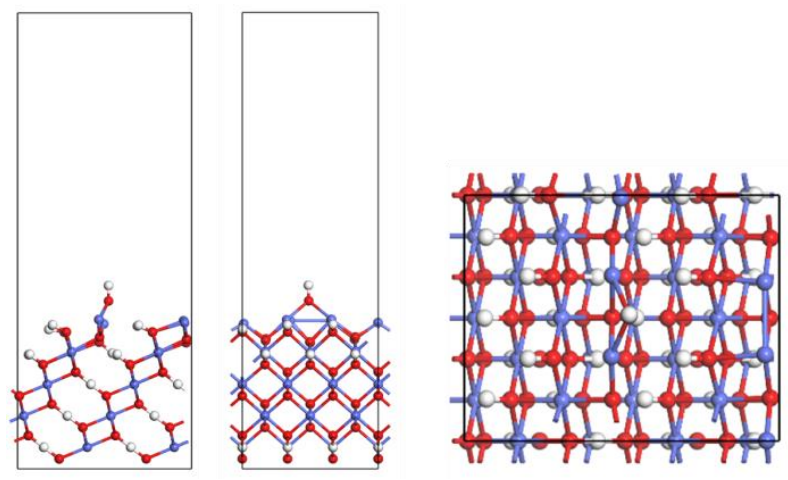

(c)
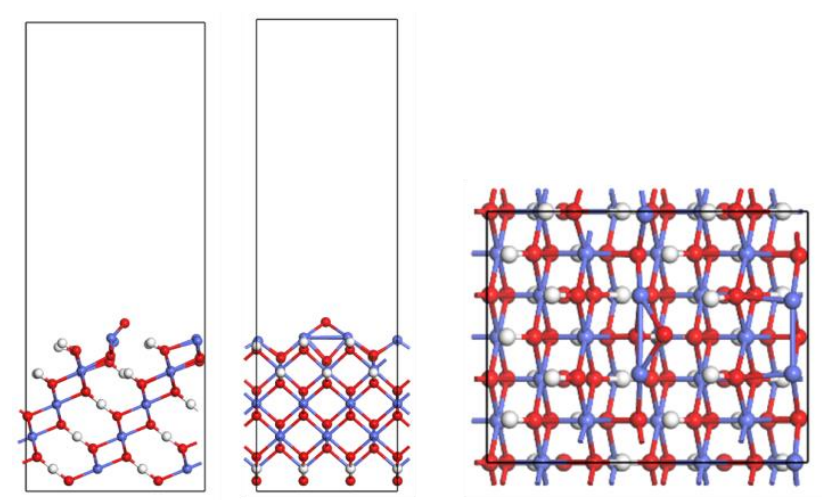

(d)
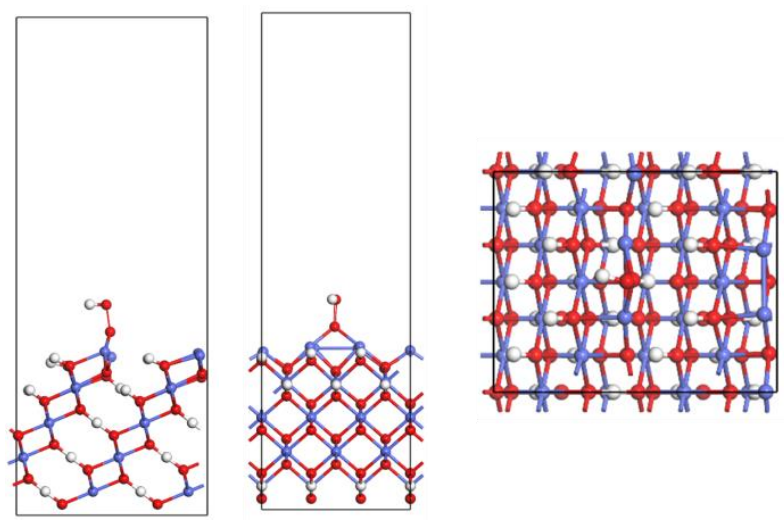

Figure S24 Optimized configurations of (a) (104) of $\mathrm{CoOOH}$ and (b) $\mathrm{OH}^{*}$, (c) $\mathrm{O}^{*}$, and (d) $\mathrm{OOH}^{*}$ species adsorbed on (104) of $\mathrm{CoOOH}$. 
Table S4 Adsorption energy of $\mathrm{OH}^{*}, \mathrm{O}^{*}, \mathrm{OOH}$ on $\mathrm{Vo}-\mathrm{CoOOH}$ and perfect $\mathrm{CoOOH}$.

Vo-CoOOH

Perfect $\mathrm{CoOOH}$

\begin{tabular}{cccc} 
Active intermediate & Absorption energy / eV & Active intermediate & Absorption energy / eV \\
\hline $\mathbf{O H}^{*}$ & -2.56 & $\mathbf{O H}^{*}$ & -4.44 \\
$\mathbf{O}^{*}$ & -3.13 & $\mathbf{O}^{*}$ & -6.25 \\
$\mathbf{O O H}^{*}$ & -1.44 & $\mathbf{O O H}^{*}$ & -2.80 \\
\hline
\end{tabular}

References

[1] R. Zhang, Y. Zhang, L. Pan, G. Shen, N. Mahmood, Y. Ma, Y. Shi, W. Jia, L. Wang, X. Zhang, W. Xu, J. Zou. ACS

Catal.2018, 8, 3803-3811.

[2] D. Wu, Y. Wei, X. Ren, X. Ji, Y. Liu, X. Guo, Z. Liu, A. Asiri, Q, Wei, X. Sun. Adv. Mater. 2018, 30, 1705366 